\title{
Implementation methods for vision related quality of life questionnaires
}

\author{
J S Wolffsohn, A L Cochrane, N A Watt
}

\begin{abstract}
Aim-To determine the most reliable and consistent method and time interval over which to implement a vision impairment quality of life assessment tool.

Methods-117 patients with low vision aged 9-101 years were assigned into three age, sex, and visual function matched groups ( $n=39$ in each) to answer the Low Vision Quality of Life (LVQOL) questionnaire by post, telephone, or in person. The LVQOL questionnaire was completed on four occasions, each separated by four weeks.

Results-Postal implementation was the most cost effective method, showed the highest internal consistency of LVQOL items, but resulted in a lower apparent quality of life score than either telephone or in-person interviews $(p<0.001)$. There was no difference in test-retest reliability between the three methods of implementation $(p=0.12)$. The profile of LVQOL scores showed a trend towards reduced quality of life scores 3 months after the baseline measures, although this was not significant.

Conclusion-Posting may be the method of choice for clinical measurement of vision related quality of life. Patients with greater visual impairment were no less likely to complete a questionnaire when implemented by post and there was no apparent bias from other people assisting them. The quality of life measure can occur at any time up to 2 months after low vision rehabilitation for the progressive nature of conditions causing low vision not to cause a decreased baseline score. The LVQOL was shown to be a highly internally consistent and reliable method for measuring quality of life in the visually impaired.

(Br f Ophthalmol 2000;84:1035-1040)
\end{abstract}

Victorian College of Optometry, University of Melbourne, Victoria, Australia J S Wolffsohn A L Cochrane N A Watt

Correspondence to: Dr J S Wolffsohn, Vision Science, Aston University, Aston Triangle, Birmingham B4 7ET, UK

j.s.w.wolffsohn@aston.ac.uk

Accepted 26 April 2000
Quality of life instruments are becoming more commonly used as researchers and managers conclude that clinical measures do not fully assess the impact and cost effectiveness of rehabilitation, treatment, or surgery. The World Health Organisation defined quality of life as "an individual's perception of their position in life in the context of the culture and value systems in which they live and in relation to their goals, expectations, standards and concerns". ${ }^{1}$ They also define health as a state of complete "physical, mental and social well being" and not merely the absence of disease or infirmity. ${ }^{2}$
Quality of life measures were initially used to determine the success of surgical interventions such as cataract surgery..$^{3-5}$ Questionnaires were later designed to measure the contentment of patients suffering from a variety of ocular diseases such as glaucoma ${ }^{167}$ and optic neuritis. ${ }^{8}$ Although quality of life questionnaires have been used in many areas of visual management, few have been designed specifically to measure the outcome of a low vision rehabilitation service. There has been little research to determine the most appropriate method of implementation for quality of life questionnaires or to establish the most suitable time interval over which to assess patients' quality of life.

There are three main ways in which a questionnaire can be implemented-by post, telephone or using in-person interviews. ${ }^{9}$ The method of implementation for a vision related questionnaire may bias the data collected. For example, with a postal questionnaire those with better vision are more likely to be able to read the questionnaire easily and respond. Mangione and colleagues found no difference in mean score for their Activities of Daily Vision Scale whether the interview was conducted in person or by telephone. ${ }^{10}$ While some vision impairment questionnaires have been designed to be administered face to face because of possible difficulties with self completion and to achieve a high response rate, others use a self completion format to facilitate postal implementation. ${ }^{11}{ }^{12}$

It is important to determine how the quality of life of a visually impaired population changes over time. Most questionnaires have had their reliability tested over a period of 7-21 days, ${ }^{11} 1314$ some even over a period as short as a few hours after the initial interview. ${ }^{15}$ However, it is likely that the answers to a short questionnaire are remembered over a short time interval, increasing apparent reliability.

To assess changes in a patient's quality of life - for example, as a result of rehabilitationbefore and after measures need to be taken. It is sensible to take the baseline (before rehabilitation) measure immediately before the rehabilitation process commences. It is, however, more complicated to determine the most appropriate time at which to measure the outcome as the causes of low vision are on the whole progressive. A comprehensive low vision rehabilitation usually involves more than one visit to a low vision clinic and best results are often achieved with interactive training over time. ${ }^{16}$ Training may occur in a clinical setting, by loaning the patient the relevant optical aids for use in the home, or by arranging home visits and varies from one low vision rehabilita- 
tion programme to another. It is possible that perceived quality of life will improve initially after low vision rehabilitation as the patient's ability to use low vision devices increases and advice given can be put into practice. Conversely, the progressive nature of a patient's visual condition might result in reduced acuity with time, making activities of daily living more difficult despite the low vision rehabilitation, leading to a reduced quality of life. It is therefore important to examine how quality of life varies with time so that measures can be taken long enough after low vision rehabilitation that the full benefits may have been received by the patient, but not so long after rehabilitation that baseline levels have fallen because of the progressive nature of low vision eye conditions.

\section{Methods}

A random sample of 150 people with low vision were recruited from the database of the Vision Australia Foundation. The subjects were asked to complete the Low Vision Quality of life (LVQOL) questionnaire which has been specifically designed to measure the quality of life of the visually impaired in a rehabilitation setting. ${ }^{17}$ The LVQOL has 25 items which examine quality of life issues caused by low vision. Each item is graded on an ordinal scale between 5 (no problem due to vision) and 1 (great difficulty due to vision). The item could also be scored as no longer possible due to vision (attributed a grade of 0 ) or as not relevant to them in their daily lives (attributed the average score of their total responses to avoid bias in the results of those who had less items relevant to them than others). A summed score is then calculated (between 0 and 125). Unlike many of the vision related quality of life assessment tools previously used with the visually impaired, the LVQOL was designed and tested using a low vision population typical of that found in developed countries. The LVQOL has only 25 items and does not take long to implement, which means it can be used in a clinical setting without placing too much additional burden on patients.

A full eye examination was performed on each subject. This included: (1) best corrected distance acuity measured with a Bailey-Lovie distance acuity chart $^{18}$; (2) contrast sensitivity measured by the Melbourne Edge Test (MET). The chart was held at $40 \mathrm{~cm}$ with a luminance of $47 \mathrm{~cd} / \mathrm{m}^{2}$ and subjects were required to identify the direction (either vertical, horizontal, sloping left or sloping right) of the edge separating the lighter from the darker half of each circle. ${ }^{19}$ The contrast difference between the circles progressively

Table 1 The post, telephone, and in person Low Vision Quality of Life questionnaire (LVQOL) implementation groups that were balanced for age, sex, distance visual acuity, contrast sensitivity, and presence of a field defect in the central $20^{\circ}$ visual field

\begin{tabular}{lllllll}
\hline $\begin{array}{l}\text { LVQOL } \\
\text { implementation } \\
\text { method }\end{array}$ & $\begin{array}{l}\text { No of } \\
\text { subjects }\end{array}$ & $\begin{array}{l}\text { Median age } \\
\text { (years) }\end{array}$ & M:F ratio & $\begin{array}{l}\text { Visual } \\
\text { acuity } \\
\text { (logMAR) }\end{array}$ & $\begin{array}{l}\text { Contrast } \\
\text { sensitivity (dB) }\end{array}$ & $\begin{array}{l}\text { Central } \\
\text { field defect } \\
\text { (\%) }\end{array}$ \\
\hline Post & 39 & $81(14)$ & $1: 1.8$ & $0.95(0.51)$ & $9.3(4.3)$ & 51 \\
Telephone & 39 & $79(18)$ & $1: 1.8$ & $0.90(0.50)$ & $9.6(5.6)$ & 59 \\
In person & 39 & $82(17)$ & $1: 1.8$ & $1.02(0.47)$ & $9.7(4.8)$ & 59 \\
\hline
\end{tabular}

Standard deviation is stated in parentheses for age, visual acuity and contrast sensitivity. decreases and threshold is reached when two successive plates are scored incorrectly (recorded in decibels); (3) examination for the presence of visual field scotomas or disruption in the central $20^{\circ}$ of vision using an Amsler chart held at $14 \mathrm{~cm}$.

The subjects were randomly assigned into three groups. The first group received the LVQOL questionnaire by post with a pre-paid addressed envelope enclosed for return of the completed version. The second group received a telephone call from one of the three authors and the LVQOL questionnaire was completed orally. The third group was visited by one of the authors in their own home and the LVQOL questionnaire was completed by an oral interview.

The second implementation of the questionnaire was conducted 4 weeks later, the third 8 weeks later, and the fourth at 12 weeks (SD 4 days) after the first completion of the LVQOL questionnaire. The same method of implementation was used on each occasion to examine whether any changes in the quality of life of the subjects occurred with time. Subjects who filled in the questionnaire by post were questioned as to whether they filled in the questionnaire themselves or whether it was filled in for them by proxy. If postal questionnaires had not been received within 1 week of their due date, subjects were telephoned to encourage them to return the completed questionnaire. They were encouraged to answer honestly and were asked to record how long it took to complete the questionnaire on each occasion.

Only those who completed the questionnaire at all four time points ( $\mathrm{n}=136,90 \%$ ) were included in the analysis. The groups were then matched for age, sex, distance acuity, contrast sensitivity, and the presence of a field defect in the central $20^{\circ}$ of visual field, resulting in each of the analysis groups having 39 subjects. Subjects had a wide range of eye conditions causing low vision and were aged between 11 and 99 years (median 80).

The project was approved by the institutional review boards of the Vision Australia Foundation and the Victoria College of Optometry.

\section{STATISTICAL ANALYSIS}

The results were entered into a spread sheet and the summed score of the LVQOL items were calculated for analysis. Analysis of variance was used to examine overall effects between groups and Tukey's pairwise multiple comparison test was used to assess individual group differences. Internal consistency between items of the LVQOL questionnaire was measured by Cronbach's $\alpha$ and reliability of repeated measures of patient's quality of life was assessed using the intraclass correlation coefficient. $^{9}$

\section{Results}

Details regarding the groups assigned to receive the LVQOL questionnaire by post, telephone, and by in person interviews are given in Table 1 . The conditions causing low 
Implementation time

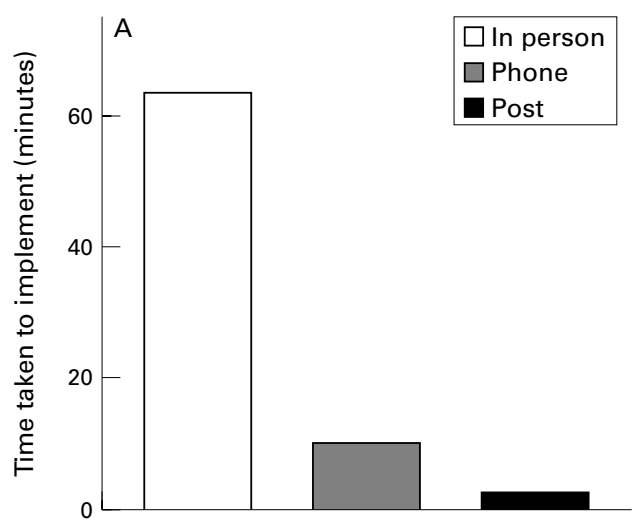

Consumable costs

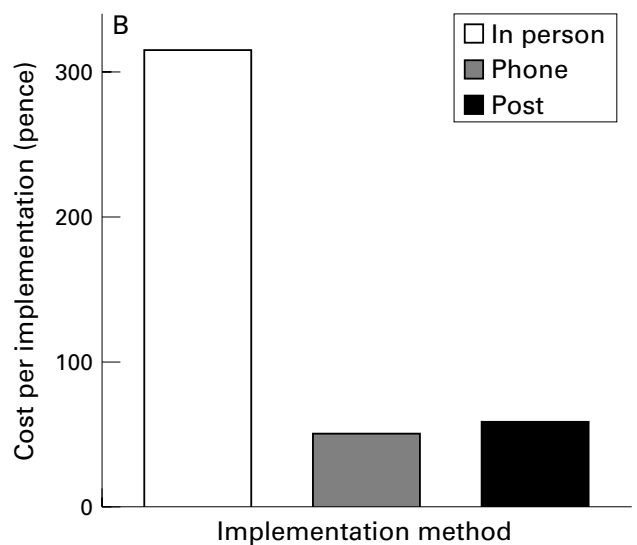

Figure 1 (A) Staff time and (B) consumable costs of implementing the LVQOL questionnaire by post $(n=39)$, telephone $(n=39)$, or in person $(n=39)$ interviews. Postal implementation was the most cost effective method.

vision in each group were approximately $40 \%$ atrophic and $25 \%$ exudative age related macula degeneration, $10 \%$ open angle glaucoma, $5 \%$ senile cataracts, and $5 \%$ diabetic retinopathy.

Of the original 150 patients with low vision who were randomly selected to take part in the study, there was no significant difference in the percentage who did not complete the four questionnaires between those who were assigned to the post $(10 \%)$, telephone $(9 \%)$, or in person $(9 \%)$ implementation groups. The main reasons for not completing the study were a decline in mental health $(2.2 \%)$, stroke
(1.6\%), moving from the state $(1.6 \%)$, and an inability for the researchers to contact the subject $(4.4 \%)$.

The cost of implementing the LVQOL questionnaire by in-person interviews in the subject's own home was found to be greater than when the implementation was by telephone interview or by post, both in staff time (Fig 1A) and consumable costs such as fuel, telephone, and postal charges (Fig 1B). The exact costing will vary from country to country and from state to state depending on the cost of staffing and local services, but the comparative cost differences are likely to remain similar. The basis of the costing is given in Table 2. Overall, postal questionnaires were found to be the most cost efficient method of implementation, costing approximately $90 \mathrm{p}$ per person (AU\$2.25), with telephone implementation costing three times more and in-person interviews costing 19 times more. If a quality of life measurement tool was implemented before and after rehabilitation, the costs will be doubled except for posting implementation where the questionnaire can be sent out with the initial appointment letter and can be returned on the patient's visit to the clinic, resulting in further cost savings compared with the other implementation methods.

The time subjects took to complete the LVQOL questionnaire was significantly shorter $(\mathrm{F}=15.3, \mathrm{p}<0.01)$ when the questionnaire was completed by telephone rather than by post or by an in-person interview (Fig 2). There was no significant difference between the time taken to complete an in-person interview and the implementation of the questionnaire by post. When the LVQOL questionnaire was implemented by post, fewer than $0.5 \%$ of the items were left incomplete, perhaps because of the relatively low time demand of the questionnaire.

Internal consistency (as measured with Cronbach's $\alpha$ ) was significantly higher ( $F=$ $15.46, p=0.001$ ) when the LVQOL questionnaire was implemented by post $(0.91(0.01))$ than by telephone $(0.81(0.01) ; \mathrm{p}<0.05)$ or in person $(0.86(0.01) ; \mathrm{p}<0.01)$. There was no significant difference in reliability of the questionnaire, measured on four occasions over a 3 month period, with the type of implementation used (visit 0.60 ; phone 0.70 ; post $0.70 ; \mathrm{F}=2.20, \mathrm{p}=0.12)$.

Table 2 Costs associated with post, telephone, and in person implementation of the Low Vision Quality of Life Questionnaire (LVQOL)

\begin{tabular}{llll}
\hline Costs & Postal implementation & Telephone implementation & In person implementation \\
\hline $\begin{array}{l}\text { Consumables } \\
\text { Materials }\end{array}$ & $\begin{array}{l}\text { Photocopying LVQOL forms onto a } \\
\text { double sided A3 sheet } \\
\text { Envelopes }\end{array}$ & & $\begin{array}{l}\text { Photocopying LVQOL forms onto } \\
\text { a double sided A3 sheet }\end{array}$ \\
& $\begin{array}{l}\text { Computer printed address labels } \\
\text { Postage }\end{array}$ & & \\
Telephone & Follow up phone call in 20\% of cases & Telephone calls to implement & Telephone calls to arrange visit \\
Travel & LVQOL & Vehicle and petrol costs \\
Time & Form and envelope preparation; & Follow up telephone call in $20 \%$ of & Telephone call administration of \\
& cases & $\begin{array}{l}\text { Form preparation } \\
\text { Phone call to arrange visit } \\
\text { Time with patient and travelling }\end{array}$ \\
& Reviewing forms and data entry & Data entry directly into computer & Reviewing forms and data entry \\
\hline
\end{tabular}

^Indicates those tasks that must be performed by a highly trained interviewer. 


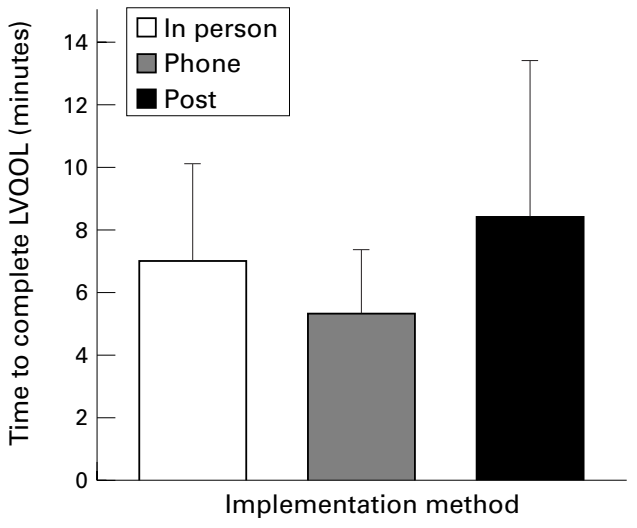

Figure 2 Time taken for visually impaired patients to complete the LVQOL questionnaire by post $(n=39)$, by telephone $(n=39)$, or by in-person $(n=39)$ interviews. Telephone implementation was significantly faster. Error bars $=1 S D$.

After summing the graded item scores, there was no significant difference between the LVQOL overall rating measure over a 3 month period after the initial measure for each of the implementation methods $(\mathrm{F}=0.64, \mathrm{p}=0.69)$. However, the mean overall rating when the

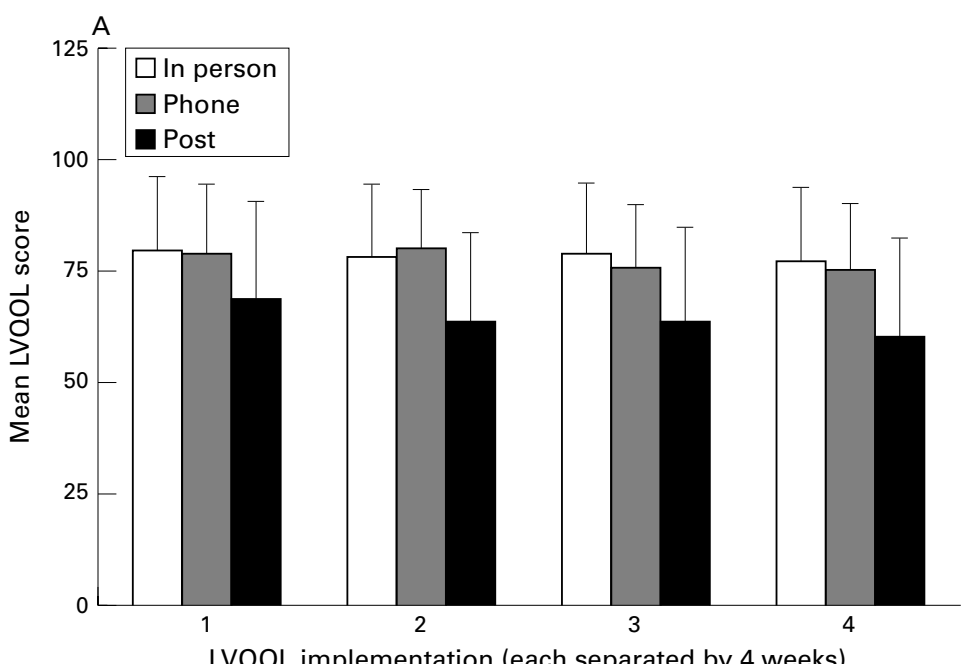

LVQOL implementation (each separated by 4 weeks)

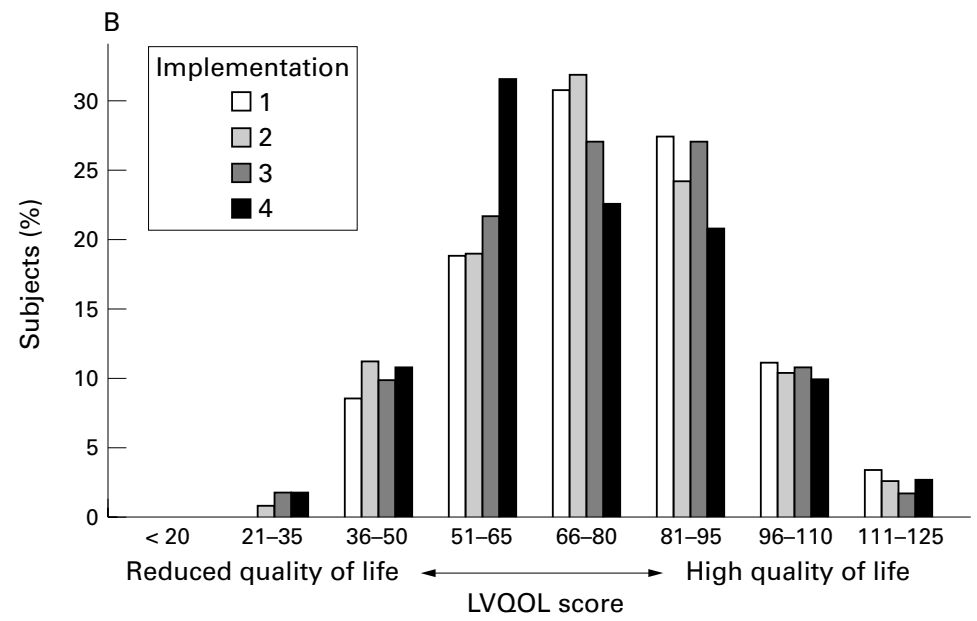

Figure 3 (A) Mean summed score for the LVQOL questionnaire over a 3 month period by post $(n=39)$, by telephone $(n=39)$, or by in-person $(n=39)$ interviews. There was no significant difference in scores over this time period, but those completing the questionnaire by post had an apparently lower quality of life. Error bars $=1 S D$. (B) Distribution of summed LVQOL scores over a 3 month period. There was a decrease in apparent LVQOL score 3 months after the initial implementation, but this was not found to be significant $(n=117)$. questionnaire was completed by post was significantly lower $(\mathrm{F}=40.97, \mathrm{p}<0.001)$ than that found with telephone or in-person interviews (Fig 3A). However, when the data for the 117 subjects were grouped together it is apparent that there is a shift towards a reduced quality of life by the third month after the initial LVQOL implementation (Fig 3B). There was no relation between the reduction in the LVQOL score and the reported duration of the condition causing low vision $(r=0.07)$.

\section{Discussion}

The purpose of this study was to examine the most appropriate and cost effective method with which to implement quality of life questionnaires to those with visual impairment. If before and after measures of quality of life are to be used to quantify changes, such as with rehabilitation or treatment, any deterioration in the quality of life of those with visual impairment over time needs to be established.

Implementing the questionnaire by post resulted in significant cost savings compared with the other implementation methods, particularly in the area of staff time. Postal questionnaires do not require experienced interviewers for implementation, so the cost of personnel time is further reduced. It might be surprising that, excluding staff time, the cost of telephone implementation was less than postal questionnaires, but postal implementation involved postage costs (including a pre-paid addressed envelope) and telephone calls to chase up any questionnaires which were not promptly returned (about $20 \%$ ). At a cost of at least $£ 1.00$ (AU\$2.40) per person (including staff time), measuring the outcome of a low vision service is not inexpensive. However, the results help to quantify the benefits of the service, increase the clinician's understanding of the patient's difficulties, and hopefully assist in securing government funding. Clinical quality of life measures will also allow the examination of different rehabilitation models, enabling new magnification aids, training, and services to be evaluated. Costs could be further reduced by using the LVQOL assessment tool on a random sample of patients attending a clinic during the year rather than with every patient or by using the tool at specific times of the year.

There was no significant difference in reliability between implementation methods, although it was slightly higher for postal and telephone implementation. Internal consistency of the questionnaire items (how homogeneous they are, tapping different aspects of the same trait) was significantly higher with postal implementation. This may be because patients are able to reference back to previous questions and can change initial gradings with this form of implementation. The internal consistency achieved with any of the implementation methods shows that the questionnaire items all assist to establish the patient's subjective vision related quality of life without having items that are redundant (due to replication of themes). ${ }^{9}$

Although the questionnaire was completed fastest by telephone, it took less than 10 
minutes to finish with each of the implementation methods. This is faster than other vision related questionnaires such as the MLVQ and the NEI-VFQ and allows the LVQOL to be used clinically without a significant burden on the patient or practitioner. ${ }^{114}$

As there was no difference in the number of subjects who did not complete the four questionnaires between each of the implementation groups, it would seem that the group receiving the questionnaire by post could find other ways in which to complete the LVQOL if their vision was not good enough to read it. The LVQOL questionnaire was printed in N24 (3M) print so that many patients with low vision could read it without the assistance of magnification aids. ${ }^{20} \mathrm{~A}$ possible bias arises from the selection of patients from a low vision clinic. All such patients will have had to respond to letters from the clinic in the past and therefore those who do not have support for reading and are unable to read moderately large print may never make it onto the clinic database. The LVQOL questionnaire is designed specifically to assess the outcome of low vision rehabilitation, so this possible bias is not of concern but it may affect the relevance of these findings to the use of vision related questionnaires implemented by post in the general population.

A lower LVQOL score (indicating a lower quality of life) consistently occurred with postal implementation of the vision related quality of life questionnaire. Patients may feel more positive with the personal contact from an eye specialist involved with telephone and in-person interviews. They may want to give a better impression of how they are coping to please the practitioner. Also, there is a level of interpretation involved with telephone and inperson interviews, as it was found that patients were keen to talk about their problems in general rather than to answer the specific question, particularly when they were required to grade individual items. Thus, postal questionnaire results may have been less biased by forcing subjects to grade items without any prompting from a researcher.

However, another alternative is that responding to the mail survey may have been a more difficult task (having to read the questionnaire, even in large type), negatively biasing postal responses. A number of subjects reported using low vision aids to complete the questionnaire and one third of the subjects who received it by post used a family member or friend to read the questionnaire items to them and to record their responses. This introduces a possible bias from the family member or friend and may have caused the consistently lower quality of life found with this method of implementation. As expected, further analysis of the results showed that those who completed the questionnaire by proxy were slightly older (mean age 83 (7) years versus 76 (13) years), had a lower visual acuity (1.25 (0.30) logMAR versus $0.80(0.53) \log$ MAR) and a reduced contrast sensitivity $(6.7$ (4.6) $\mathrm{dB}$ versus 11.1 (3.5) $\mathrm{dB}$ ) compared with those who could complete the questionnaire with no assistance.
Those who required assistance also had a reduced quality of life (73.8 (19.3) versus 94.7 (13.8) LVQOL score) compared with those who could complete the questionnaire with no assistance. Although a poorer quality of life is expected with greater visual impairment, the lower score found with postal implementation of a quality of life questionnaire compared with telephone or in-person implementation could also reflect a negative bias from the person assisting the completion of the questionnaire influencing the result. However, when the results of those who received the LVQOL questionnaire by post and were able to complete it without assistance were compared with a visual acuity matched group of visually impaired subjects who completed the questionnaire by telephone, their quality of life was again found to be consistently reduced. This bias therefore has a minor, if any, effect on the LVQOL score. All the patients who had assistance in completing the questionnaire reported that the same person assisted them on each occasion, so any bias would be consistent and the change in LVQOL score between before and after measures would be unaffected. Postal implementation of the LVQOL questionnaire therefore appears to be a valid clinical measure of a patient's vision related quality of life, without fear of bias and at a substantial cost saving compared with telephone or in-person implementation methods. However, the quality of life scores measured using postal questionnaires are not comparable with data collected using other forms of implementation and studies using several forms of implementation must be analysed with caution.

For any of the implementation methods, the summed scores from the LVQOL appeared to stay constant over a 2 month period following baseline measurement. After this time the progressive nature of conditions causing low vision would appear to reduce the quality of life of the visually impaired. It is possible that an initial learning effect in completing the LVQOL questionnaire occurred which improved the results with time, masking an actual decrease in LVQOL scores over the first 2 months. This would seem unlikely as there was a 1 month time period between questionnaires. Ideally, the study would have examined 12 groups, four completing the quality of life questionnaire by each implementation method, one group at each time period after low vision rehabilitation. However, reliably matching each group for all the factors likely to play a part in the overall quality of life score would be difficult.

As the vision related quality of life score remains essentially constant over a 2 month period, even with the progressive nature of the diseases causing low vision, the results of questionnaires assessed at any time in this period are comparable. After this period the quality of life of the visually impaired appears to decrease, showing that the LVQOL questionnaire is sensitive to change. It would be sensible to leave final assessment of the quality of life until after any return visits to the clinic or continued training, as long as this is within a 2 month period after the baseline measure, as 
the full benefit of the services received may not be experienced by some individuals before this time. The data from this study could be used as a control, no treatment group in further studies examining the effect of low vision rehabilitation on the quality of life of the visually impaired as measured using the LVQOL.

In conclusion, the results of this study suggest that postal implementation is a cost effective, reliable, and valid way of implementing vision related quality of life questionnaires in a clinical setting. However, the grading of patient's quality of life will be lower using postal questionnaires than with telephone and in-person interviews. There is no bias resulting from patients with poorer vision being less likely to complete the questionnaire with postal implementation or from them using other people to assist them to complete it. Finally, implementation should occur before and up to two months after low vision rehabilitation for the outcomes of the service to be assessed.

Funding: This research was funded by a collaborative grant between the Victorian College of Optometry and the Vision Australia Foundation.

1 Parrish RK. Visual impairment, visual functioning, and quality of life assessments in patients with glaucoma. Trans Am Ophthalmol Soc 1996;19:919-1028.

2 Ellwein LB, Fletcher A, Negrel AD, et al. Quality of life assessment in blindness prevention interventions. Int $\mathrm{Oph}$ thalmol 1995;18:263-8.

3 Elliott DB, Hurst MA, Weatherill J. Comparing clinical tests of visual function in cataract with the patient's perceived visual disability. Eye 1990;4:712-7.

4 Abrahamsson $\mathrm{M}$, Carlsson B, Tornqvist M, et al. Changes of visual function and visual ability in daily life following cataract surgery. Acta Ophthalmol (Scand) 1996;74:69-73.
5 Lundstrom $\mathrm{M}$, Roos $\mathrm{P}$, Jenson $\mathrm{S}$, et al. Catquest questionnaire for use in cataract surgery care: description, validity, and reliability. $\mathcal{F}$ Cataract Refract Surg 1997;23:1226-36.

6 Ross JE, Bron AJ, Clarke DD. Contrast sensitivity and visual disability in chronic simple glaucoma. $\mathrm{Br} \mathcal{F}$ Ophthalmol 1984;68:821-7.

7 Gutierrez P, Wilson R, Johnston C, et al. Influence of glaucomatous visual field loss on health-related quality of life. Arch Ophthalmol 1997;115:777-84.

8 Cleary PA, Beck RW, Bourque LB, et al. Visual symptoms after optic neuritis: results from the optic neuritis treatment trial. f Neuro-Ophthalmol 1997;17:18-28.

9 Streiner DL, Norman GR. In: Health measurement scales. A practical guide to their development and use. Oxford: Oxford Medical Publications, 1996.

10 Magione CM, Phillips RS, Seddon JM, et al. Development of the 'activities of daily vision scale'. A measure of visual functional status. Med Care 1992;30:1111-26.

11 Harper R, Doorduyn K, Reeves B, et al. Evaluating the outcomes of low vision rehabilitation. Ophthal Physiol Opt 1999;19:3-11.

12 Frost NA, Sparrow JM, Durant JS, et al. Development of a questionnaire for measurement of vision-related quality of life. Ophthal Epidemiol 1998;5:185-210.

13 Bullimore MA., Raasch TV, Cutter GC, et al. Quality of life assessment in a low vision population. Invest Ophthalmol Vis Sci 1997;38:S710.

14 Mangione CM, Lee PP, Pitts J, et al. Psychometric properties of the National Eye Institute Visual Function Questionnaire (NEI-VFQ). Arch Ophthalmol 1998;116: 1496-504.

15 Carta A, Braccio L, Belpoliti M, et al. Self-assessment of the quality of vision: association of questionnaire score with objective clinical tests. Curr Eye Res 1998;17:506-11.

16 Faubert J, Overbury O. Active-passive paradigm in assessing CCTV-aided reading. Am f Optom Physiol Opt 1987;64:23-

17 Wolffsohn JS, Cochrane AL. The Low Vision Quality of Life (LVOOL) assessment tool. How to implement a low vision questionnaire. Optom Vis Sci 1998;75:S27.

18 Bailey IL, Lovie JE. New design principles for visual acuity letter charts. Am f Optom Physiol Opt 1976;53: $740-5$.

19 Greeves AL, Cole BL, Jacobs RJ. Reliability and validity of simple photographic plate tests of contrast sensitivity. Am $\mathcal{F}$ Optom Physiol Opt 1987;64:832-41

20 Leat SJ, Rumney NJ. The experience of a university-based low vision clinic. Ophthal Physiol Opt 1990;10:8-15. 